\title{
Antimicrobial treatment for urinary tract infections
}

\author{
$\mathrm{K}$ Verrier Jones
}

The aims of treatment

One of the aims of treatment of urinary tract infection is to relieve distressing symptoms as quickly as possible. Another is to prevent recurrent infections in those children who experience frequently recurring symptomatic urinary tract infection. A third objective is to prevent infections in children who are deemed to be at greatest risk of developing reflux nephropathy or other forms of renal damage-that is, the very young child with vesicoureteric reflux. The management of urinary tract infection in childhood has been reviewed extensively by Jodal and Winberg, ${ }^{1}$ McCracken, ${ }^{2}$ and White. ${ }^{3}$

Before the start of treatment it is essential that the urine is examined microscopically and sent to the laboratory for culture, sensitivity, and colony counting. In infants suprapubic aspiration of urine under ultrasound control is the method of choice and where bag collection is used there will inevitably be a significant contamination rate with an appreciable number of false negative and false positive results leading in some cases to a delay in the onset of treatment and in others to unnecessary investigation. The risk of diagnostic error can be reduced by collecting two bag or clean catch samples and by the use of microscopy at the bedside. It is unacceptable to wait $\mathbf{4 8}$ hours for the results of culture and sensitivity before starting treatment in an infant or acutely symptomatic child. Paediatricians should become accustomed to making the diagnosis on the presence of white cells and organisms on direct microscopy of the unspun urine. As urinary tract infection in infancy rarely presents with symptoms localised to the urinary tract, ${ }^{4}$ and delay in treatment may result in permanent renal damage ${ }^{56}$ especially in the youngest children, ${ }^{7}$ every sick infant needs urgent urine examination.

While management of the acute or presenting urinary tract infection is relatively straightforward, the long term management of recurrent urinary tract infections, mainly in girls, and the use of prophylactic antibiotics for the prevention of reflux nephropathy is both complex and controversial. It is intimately connected with other aspects of long term management and investigation, and by the natural history and epidemiology of urinary tract infection.

Swedish data suggest that the commonest age for the first urinary tract infection is the first year of life ${ }^{8}$ and that the risk in childhood decreases thereafter. Although the incidence is higher in boys in the first month, by six months urinary tract infections are more prominent in girls. Recurrent urinary tract infections occur in more than half the girls affected, but are uncommon in boys. Common host factors that predispose to renal damage are young age, ${ }^{9}$ vesicouretric reflux, ${ }^{4}$ and delay in onset of treatment, although the presence of obstruction, neurogenic bladder, and stones are less common but equally important and potentially treatable. Bacterial virulence factors are also likely to influence the development of symptoms and reflux nephropathy.

\section{Treatment of the acute infection}

Treatment should be preceeded by a thorough history and physical examination and special note made of urinary symptoms and recent antibiotic treatment. Examination should include an assessment of the child's general well being, degree of toxicity, and dehydration, if the child is acutely unwell, as well as the presence of a palpable bladder or kidneys and neurological signs in the lower limbs. In the acutely sick infant or child it is important to start to correct shock, dehydration, and electrolyte imbalance as soon as possible and antipyretics should be given to febrile children. In this acute situation the use of intravenous aminoglycosides is indicated and can be expected to result in a brisk clinical response, although the standard dose will need to be modified in those with acute or chronic impairment of renal function and levels monitored. Alternative, but less satisfactory, broad spectrum intravenous antibiotics are amoxycillin, the cephalosporins, amoxycillin and clavulanic acid in combinations (Augmentin, Beecham), azlocillin, and the aminoquinolones. The aminoquinolones are not generally recommended for children, however, and should be reserved for those cases where the more well tried antibiotics are ineffective, such as multiply resistant pseudomonas infections. ${ }^{10}$ In very severe and complicated infections intravenous treatment may be continued for five to 10 days, but more often the child is considerably better by 48 hours and a suitable oral antibiotic can be chosen according to the sensitivity reported by the laboratory. A one week course of oral treatment is usually sufficient and can be completed at home before changing to long term, low dose prophylaxis, which should be continued until investigation of the urinary tract has been completed, all the risk factors for development of reflux nephropathy have been 
assessed and long term management plans have been made. ${ }^{2}$

Most children, including some infants and children with systemic upset, will be only mildly unwell and able to tolerate oral fluids and oral antibiotics. Trimethoprim, amoxycillin, and clavulanic acid in combination are potent, effective broad spectrum antibiotics that give good results in most cases. Amoxycillin alone is likely to be effective against only $60-70 \%$ of Escherichia coli and cephalosporins are expensive and have not been as effective as other drugs in clinical trials. If the organism is sensitive to amoxycillin, however, this is an excellent and safe drug, which should be used in preference to the combination with clavulanic acid to reduce the risk of bacterial resistance to the newer drug. Five to seven days' treatment is effective in most cases and prolonged courses increase the risk of diarrhoea and thrush.

If there is no clinical improvement within 48 hours either the organism is not sensitive to the antibiotic chosen, or there is an underlying urological problem, such as obstruction. The antibiotic sensitivity should be reviewed, urgent ultrasound of the kidneys and urinary tract arranged and intravenous treatment considered if only oral treatment has been used so far. If obstruction is present a surgical opinion should be sought and drainage by a nephrostomy, or a suprapubic or bladder catheter, arranged as soon as possible.

In many children the symptoms of urinary tract infection are fairly trivial with symptoms largely confined to the lower urinary tract and little systemic upset. Even in this situation when the child is unlikely to require hospital admission there is no justification for a delay in treatment. Symptomatic lower urinary tract infections are generally associated with a significant increase in urinary white cells and can be easily diagnosed microscopically and subsequently confirmed on culture. In addition to the oral antimicrobial drugs already mentioned nalidixic acid and nitrofurantoin are excellent urinary antiseptics with a broad range of activity against Gram negative organisms. Good tissue concentrations are not achieved, however, and they should not be used in systemically ill children or in those in whom acute pyelonephritis is suspected or in children with renal failure.

\section{Prophylaxis}

Prophylactic treatment is the use of a drug in a high risk situation to reduce the chance of subsequent infection. It is the duty of the paediatrician to anticipate those situations where this may be of value and ensure that appropriate treatment is given. Catheterisation of the urinary tract for any reason carries a high risk of introducing bacteria at the time of the procedure. ${ }^{11}$ In paediatric wards this occurs most commonly at the time of a micturating cystogram and unless prophylaxis is given routinely in this situation, the children who are most at risk of acquired renal damage will be put at increased risk by this procedure. Our policy is to give trimethoprim in full dosage for $\mathbf{4 8}$ hours from the time of catheterisation. Children who are undergoing urological instrumentation of the urinary tract receive a single intravenous injection of an aminoglycoside in full dosage, whatever their level of renal function. Before and during elective surgery on the infected urinary tract a full course of an appropriate antibiotic, usually an aminoglycoside or azlocillin or ciprofloxacin, is used to reduce the risk of Gram negative septicaemia. This is because a child with an abnormal urinary tract who has had several courses of treatment previously is likely to have multiply resistant organisms including proteus and pseudomonas, and close cooperation with a microbiologist is essential.

It is now clear that approximately $50 \%$ of infants born into families where a first degree relative has vesicoureteric reflux or reflux nephropathy also have vesicoureteric reflux themselves, although in most it is grade I or II (Medical Research Council grading). ${ }^{12}$ In this group of infants, neither the natural history nor the benefits and risks of prophylaxis or other modes of intervention have been evaluated. Clearly this group of infants poses new questions on the role of prophylactic treatment in relation to sterile vesicoureteric reflux and the prevention of reflux nephropathy as do those children with vesicoureteric reflux detected as a result of antenatal ultrasound examination. ${ }^{13}$

Most often prophylaxis is used to prevent reinfection after the first, presenting infection, because there is deemed to be an appreciable risk of progressive renal damage, which occurs mainly in children under 5 years of age with vesicoureteric reflux. The development of reflux nephropathy after the age of 5 is relatively uncommon, although some cases have been reported, mainly when severe vesicoureteric reflux has been present and there has been a delay in the onset of treatment. ${ }^{14}$ In some groups of children with established reflux nephropathy who have been followed for prolonged periods new scars have developed in a minority whether or not long term low lose prophylaxis has been used. ${ }^{15} 16$ The value of prolonged treatment in teenage girls is in doubt, unless they have frequently recurring symptoms which cannot be managed by other means. The risk of pregnancy increases significantly after 16 and it is theoretically undesirable for these girls to conceive while taking medication of doubtful value, particularly folate antagonists such as trimethoprim. In reality teenage girls are poorly motivated to take prophylaxis unless they have had very troublesome symptoms and prefer short courses of treatment when their symptoms flare up.

The drugs that have been shown to be effective in preventing reinfection are nitrofurantoin ${ }^{17}$ and trimethoprim. ${ }^{18}$ They are both used in doses of $1-2 \mathrm{mg} / \mathrm{kg} /$ day given usually as a nightly bolus; this is well below the normal therapeutic dose. Side effects have been relatively uncommon with both these drugs, but recently there has been some anxiety about the small risk of pulmonary and hepatic fibrosis in children taking nitrofurantoin for prolonged periods. ${ }^{19}$ Amoxycillin, cephalosporins, and nalidixic acid are relatively ineffective as prophylactic agents probably because the bowel 
soon becomes colonised with $E$ coli resistant to the drug taken. ${ }^{20}$ Reinfection of the urinary tract, when it occurs, is likely to be with a resistant organism. Co-trimoxazole is also effective as a prophylactic agent but the greater risk of side effects and complications from the sulphamethoxasole component of this trimethoprim combination have lead to a reduction in the use of this drug in the long term management of urinary tract infection in childhood. ${ }^{21}$

Single dose and short course treatment Single dose treatment and very short course treatment is attractive because it is associated with improved compliance and reduced side effects and cost, and it was used successfully in adolescent girls by Fine and Jacobson. ${ }^{22}$ There is evidence to suggest that those who have an early recurrence of infection may have upper tract infection or underlying abnormality. It has been suggested in adults that single dose treatment could be used as a method of screening for those who need further investigation.

In a randomised controlled study in children and adults Bailey and Abbott showed that the cure rate after a single dose of amoxycillin was as effective as a one week course. ${ }^{23}$ Others, however, have found that early recurrence after 10 days was more likely to occur after single dose treatment than a one week course, although most recurrences were asymptomatic. ${ }^{24}$ It would be prudent therefore to use single dose treatment in girls over 5 years of age with grossly normal urinary tracts in whom the risk of progressive damage is minimal, but to treat younger children and those with abnormal urinary tracts, systemic, or upper tract symptoms with longer courses.

\section{When not to treat}

There are now a number of long term follow up studies in schoolgirls with asymptomatic bacteriuria that show that this is a benign condition in girls with radiologically normal kidneys over 4 years of age even if vesicoureteric reflux is present. ${ }^{1516}$ Reflux nephropathy once established progresses very slowly in a small proportion of cases and usually when vesicoureteric reflux is present and reinfection occurs. There is no clear evidence that long term low dose prophylaxis is superior to no treatment in asymptomatic children, however, as reinfection with new organisms may be more damaging to the kidney than continuous infection with an organism of low pathogenicity. ${ }^{25}$ In girls the natural history is for reinfection to occur in a high proportion after treatment has been discontinued. Conventional courses of treatment are doomed to failure in a large proportion of girls, therefore, and long term low dose prophylaxis is less successful in asymptomatic infection because of poor compliance and lack of motivation. In boys conventional courses may be used effectively as reinfection is rarely a problem except in the grossly abnormal urinary tract.

The grossly abnormal urinary tract Recurrent asymptomatic infection is relatively common in children with neurogenic bladders or where there is gross dilatation or stasis of the urinary tract, a ureterostomy, after bladder reconstruction, or renal calculi, and in those children using self intermittent catheterisation. It is usual to treat the infection when it first comes to medical attention, particularly in children under 2 years, but infections that recur after three or four courses of appropriate antibiotic treatment in the absence of appreciable symptoms are likely to continue to recur after further courses. The organisms may become increasingly difficult to treat, however, because of acquired antibiotic resistance. It is important to ensure that proper surgical management has been carried out-for example, removal of all urinary calculi, relief of obstruction, and proper management of the neurogenic bladder. Although prophylaxis is helpful in some cases, repeated breakthrough infections often become a problem in these children. In this situation it is best to leave the infection untreated unless symptoms become troublesome or surgery is planned. The child can then be given a five to 10 day course of appropriate treatment, until there is clinical improvement, or surgery has been completed. The choice of antibiotic may be difficult and these children with multiply resistant organisms such as proteus, pseudomonas, and $E$ coli usually require treatment with expensive newer intravenous antibiotics rather than first line drugs.

Dr Verrier Jones is the Laura Ashley senior lecturer in paediatric nephrology.

1 Jodal U, Winberg J. Management of children with unobstructed urinary tract infection. Pediatric Nephrology 1987; 1:647-56.

2 McCracken GH. Diagnosis and management of acute urinary tract infections in infants and children. Pediatr Infect Dis tract infections in

3 White RHR. Management of urinary tract infection. Arch Dis Child 1987;62:421-7.

4 Smellie JM, Hodson CJ, Edwards D. Clinical and radiological features of urinary infection in childhood. $\mathrm{Br} \mathrm{Med} \mathcal{F}$ 1964;ii:1222-6.

5 Slotki IN, Asscher AW. Prevention of scarring in experimental pyelonephritis in the rat by early antibiotic therapy. Nephron 1982;30:262-8.

6 Ransley PG, Risdon RA. Reflux nephropathy: effects of antimicrobial therapy on the evolution of the early pyelonephritic scar. Kidney Int 1981;20:733-42.

7 Berg U, Johansson SB. Age as a main determinant of renal functional damage in urinary tract infection. Arch Dis Child 1983;58:963-9.

8 Winberg J, Andersen HJ, Bergstrom T, Jacobsson B, Larson H, Lincoln K. Epidemiology of symptomatic urinary tract infection in childhood. Acta Paediatr Scand 1974; ary tract infection in
63(suppl 252):1-20.

9 Winberg J. Clinical pyelonephritis and focal renal scarring. A selected review of pathogenesis, prevention and prognosis. selected review of pathogenesis, prevention
Peditr Clin North Am 1982;29:801-42.

10 Anonymous. Ciprofloxacin: an important new antimicrobial. Drug Ther Bull 1987;25:69-72.

11 Hallett RJ, Pead L, Maskell R. Urinary infection in boys. A three year prospective study. Lancet 1976;ii:1107-10.

12 Aggarwal VK, Verrier-Jones K. Vesicoureteric reflux: screening of first degree relatives. Arch Dis Child 1989; 64:1538-41.

13 White RHR. Fetal uropathy: conservative management is best. Br Med F 1989;298:1408-9.

14 Smellie JM, Ransley PG, Normand ICS, Prescod N, Edwards D. Development of new renal scars: a collaborative study. Br Med f 1985;290:1957-60.

15 Newcastle Covert Bacteriuria Research Group. Covert bacteriuria in schoolgirls in Newcastle on Tyne: a five year follow up. Arch Dis Child 1981;56:585-92.

16 Cardiff Oxford Bacteriuria Study Group. Sequelae of covert bacteriuria in school girls: a four year follow up study.

17 Normand ICS, Smellie JM. Prolonged maintenance chemotherapy in the management of urinary tract infecchemotherapy in the management
tion. $B r$ Med $\mathcal{F}$ 1965; i:1023-6.

18 Smellie JM, Katz G, Gruneberg RN. Controlled trial of prophylactic treatment in childhood urinary-tract infection. Lancet 1978;ii:175-8. 
19 Coraggio MJ, Cross TP, Roscelli JD. Nitrofurantoin toxicity in children. Pediatr Infect Dis 1989;8:163-6.

20 Winberg J, Berstrom T, Lidin-Janson G, Lincoln K. Treatment trials in urinary tract infection (UTI) with special reference to the effect of antimicrobials on the fecal and periurethral flora. Clin Nephrol 1973;1:142-8.

21 Anonymous. Co-trimoxazole, or just trimethoprim? Drug Ther Bull 1986;24:17-9.

22 Fine JS, Jacobson MS. Single dose versus conventional therapy of urinary tract infections in female adolescents. Pediatrics 1985:75:916-20.
23 Bailey RR, Abbott GD. Treatment of urinary tract infection with a single dose of àmoxycillin. Nephron 1977;18: 316-20

24 Madrigal G, Odio CM, Mohs E, Guevara J, McCracken GH. Single dose antibiotic therapy is not as effective as conventional regimens for management of acute urinary tract infections in children. Pediatr Infect Dis 1989;7:316-9.

25 Olling S, Verrier Jones K, Mackenzie R, Verrier Jones ER, Hanson LA, Asscher AW. A four year follow up of schoolgirls with untreated covert bacteriuria: bacteriological aspects. Clin Nephrol 1981;16:169-71. 